\title{
ESTRATÉGIAS DE AQUISIÇÃO DE VOCABULÁRIO ADOTADAS POR ALUNOS DE LETRAS NA APRENDIZAGEM DE INGLÊS COMO LÍNGUA ESTRANGEIRA
}

\author{
Ariane Peronio MARIA ${ }^{1}$ \\ Gabriela Quatrin MARZARI ${ }^{2}$
}

RESUMO: neste estudo pretende-se verificar quais estratégias de aquisição de vocabulário são mais frequentemente adotadas por aprendizes de Inglês como Língua Estrangeira e, portanto, mais eficientes do ponto de vista metodológico. Primeiramente, faz-se uma retomada das diferentes metodologias de ensino de Língua Inglesa, tendo como foco as principais estratégias de aquisição de vocabulário. A seguir, analisa-se o livro didático American English File 2, adotado para alunos do terceiro semestre do Curso de Letras: Português e Inglês do Centro Universitário Franciscano de Santa Maria/RS. A fim de verificar quais são as estratégias mais recorrentes para o ensino de vocabulário aos aprendizes da língua alvo, são analisadas cinco unidades do livro didático citado. Por fim, é aplicado um questionário aos alunos, com o objetivo de conferir se as estratégias que eles utilizam vão ao encontro das estratégias trabalhadas no livro didático analisado. Os resultados obtidos demonstraram que, embora no livro didático a maioria das atividades voltadas à aquisição de vocabulário faça uso da linguagem de forma descontextualizada, os aprendizes identificaram a leitura - atividade que faz uso da língua de forma contextualizada - como sendo a melhor estratégia para a aprendizagem de novos vocábulos na língua alvo.

Palavras-chave: Ensino de Inglês como Língua Estrangeira. Aquisição de vocabulário. Estratégias.

\begin{abstract}
: this study aims to determine which vocabulary acquisition strategies are more often adopted by learners of English as a Foreign Language and therefore more efficient in terms of methodology. First, different methodologies of teaching the English language are taken into consideration, focusing on the key strategies of vocabulary acquisition. Then, the textbook American English File 2, which is adopted by students of the third semester of Languages: English and Portuguese at the Franciscan University in the city of Santa Maria/RS, is analyzed. In order to determine which strategies are usually adopted to teach vocabulary to the English language learners, five units of the textbook are analyzed. Finally, a questionnaire is applied to the students, in order to check whether the strategies they say they apply to learn new words are the same strategies explored by the textbook being analyzed. The results showed that, although most of the activities found in the textbook focus on vocabulary acquisition without considering language in use, the learners identified reading - an activity that makes use of language in context - as the best strategy for learning new vocabulary in the target language.
\end{abstract}

Keywords: English as a Foreign Language. Vocabulary acquisition. Strategies. 


\section{Introdução}

Pesquisas sobre aquisição de vocabulário, realizadas por Nation (2003), Zimmerman (1997) e Paiva (2004), têm demonstrado que esta é uma questão bastante pertinente entre professores e estudiosos da área, uma vez que o nível de proficiência em vocabulário por parte do aprendiz é, na maioria das vezes, determinante para o sucesso de seu desempenho na língua alvo. Além disso, considerando-se a formação de futuros professores de línguas estrangeiras, compreender o processo de aquisição de vocabulário é necessário para ensiná-lo de forma efetiva, permitindo aos atuais aprendizes o desenvolvimento de competências profissionais específicas, dentre elas, a linguístico-comunicativa ${ }^{3}$.

Logo, tendo em vista a relevância do assunto para a formação de futuros professores de Letras-Inglês, a presente pesquisa visa a fazer um apanhado teórico sobre as diferentes estratégias de aquisição de vocabulário em Língua Inglesa, considerando diversos métodos e concepções de ensino-aprendizagem. Além disso, com base na bibliografia consultada, é feita uma análise do livro didático adotado pelos acadêmicos do terceiro semestre do Curso de Letras: Português e Inglês do Centro Universitário Franciscano, para verificar quais estratégias de aquisição de vocabulário em Língua Inglesa são recorrentes nesse material. A seguir, é aplicado um questionário aos participantes deste estudo, para verificar como o vocabulário da língua alvo tem sido adquirido por eles ao longo do processo de aprendizagem.

Por meio desta investigação, pretende-se analisar quais são as estratégias mais utilizadas pelos aprendizes de Inglês como Língua Estrangeira, futuros professores da língua alvo, com o intuito de compartilhar os resultados obtidos ao longo desta investigação com outros profissionais da área que também se interessam pelo tema aquisição de vocabulário em línguas estrangeiras. A pesquisa torna-se relevante na medida em que muitos aprendizes de Inglês como Língua Estrangeira buscam respostas para essa temática. É justamente considerando-se a necessidade de compreender como ocorre o processo de aquisição de vocabulário que, ao longo deste estudo, são apontadas algumas das estratégias de aquisição mais recorrentes e, aparentemente eficazes, durante os processos de ensino e aprendizagem da Língua Inglesa na sala de aula.

\section{Aquisição de vocabulário segundo os diferentes métodos de ensino}


A aquisição de vocabulário é um tema que sempre foi de grande preocupação em se tratando do processo de aquisição de uma segunda língua. Neste artigo, é apresentada uma breve revisão dos métodos de aquisição da linguagem ao longo da história, bem como maneiras de se apresentar e testar o novo vocabulário trabalhado nas aulas de Língua Inglesa.

Antes disso, é necessário mencionar que a escolha dos itens lexicais que são trabalhados deve ir ao encontro das necessidades dos aprendizes e também levar em consideração a relevância desses itens para a sua realidade. Com base nessa afirmação, a aula deve ser preparada considerando-se o uso frequente ou recorrente dessas palavras, o que é defendido por Nation (2003). Mas, para Brown (2001), essas palavras devem ser sempre contextualizadas e significativas para que o aprendiz se aproprie do novo vocabulário e realmente o adquira.

Quando alguém começa o estudo de uma segunda língua, o maior objetivo é desenvolver competência comunicativa nessa língua. Por essa razão, é importante considerar que a gramática e o vocabulário constituem grande parte da estrutura de uma língua, enfim, o que dará base para a performance deste aluno.

O que acontece é que, geralmente, aprendizes de Língua Inglesa com uma boa base gramatical, que conseguem se comunicar fluentemente, falam sobre o passado, presente e futuro, têm dificuldade em manter uma conversa longa pela "falta" de vocabulário. A esse respeito, McCarthy (1990) afirma que não importa quão bem um aluno aprende gramática, não importa quão bem sucedido ele é na pronúncia, sem palavras para expressar um grande leque de significados, a comunicação na L2 simplesmente não acontece de forma significativa. E é papel do professor oferecer ao aluno algumas estratégias de aquisição de vocabulário, bem como preparar suas aulas ou unidades, a fim de melhor trabalhar essas estratégias.

De acordo com Ur (1991, p. 61), o ensino de vocabulário envolve o ensino de pronúncia, grafia, gramática, colocação, significado e formação da palavra. No entanto, antes de discutir a escolha do vocabulário a ser trabalhado em sala de aula, algumas tendências históricas sobre a aquisição da segunda língua são apresentadas, conforme retomadas e descritas por Zimmerman (1997) e Paiva (2004):

- Método de Gramática e Tradução (The Grammar Translation Method): foi desenvolvido no final do século XVIII e seu principal objetivo era preparar os alunos para ler e escrever materiais clássicos e, além disso, aprová-los em provas e exames 
padronizados. O vocabulário era ensinado por meio de listas de palavras com as respectivas traduções.

- Movimento da Reforma (The Reform Movement): este método enfatiza a língua falada e o treino da fonética e foi desenvolvido nos anos 80 na Inglaterra, pelo linguista Henry Sweet. Mediante este, se estabeleceu um sistema para ensinar vocabulário que começaria pelo estágio mecânico - estudo da fonética e transcrições passaria pelo estágio gramatical - estudo da gramática e vocabulário básico - e chegaria ao estágio idiomático (aquisição do vocabulário em alto nível). Os próximos dois estágios, literacy and archaic (literário e arcaico), visavam a estudar a filologia e eram estudados a nível universitário. É importante mencionar que as aulas de Sweet eram baseadas em prática controlada da língua. Além disso, listas de palavras eram evitadas, já que para ele "although language is made up of words, we do not speak in words, but in sentences (...)" ${ }^{4}$. Logo, para que haja comunicação, é necessário que se estabeleça uma relação lógica entre as palavras, formando desse modo sentenças dentro de determinado contexto.

- Método Direto (The Direct Method): a principal característica deste método é não usar a tradução quando se trabalha o vocabulário. Foi desenvolvido por Sauveur nos Estados Unidos por volta de 1860, tornou-se famoso e ainda é o método utilizado pelas escolas Berlitz. No Método Direto, afirmava-se que "interaction was at a heart of natural language acquisition ", e vocabulário e frases do dia-a-dia são usadas. A leitura é ensinada ao longo do curso e desenvolvida por meio da prática e da fala. $\mathrm{O}$ vocabulário concreto é explicado por meio de figuras rotuladas e demonstrações, enquanto que o vocabulário abstrato, por meio de associações de ideias. A principal crítica contra este método é que ele simplificaria as similaridades entre a língua mãe e a língua estrangeira e também a falta de consideração da prática do mesmo nas escolas públicas. Trata-se de uma utopia, já que é impossível ensinar uma LE sem buscar a materna, no contexto escolar. (RICHARDS; RODGERS, 1986 apud ZIMMERMAN, 1997, p. 10).

- Método de Leitura/Método do Ensino de Línguas Situacional (The Reading Method/ Situational Language Teaching): foi criado entre as décadas de 20 e 30, nos Estados Unidos e na Grã-Bretanha. O maior objetivo deste método é desenvolver a leitura, já que o aprimoramento do vocabulário implica na melhoria dessa habilidade. Assim como Nation (2003), West (apud ZIMMERMAN, 1997) acreditava que era 
recomendável usar listas de palavras de alta frequência para selecionar e ordenar o vocabulário a ser ensinado nas diferentes instâncias de ensino. "For the first time, vocabulary was considered one of the most important aspects of second language learning and a priority was placed on developing a scientific and rational basis for selecting the vocabulary content of language courses". (WEST apud ZIMMERMAN, 1997, p.10).

- Método Audiolingual (The Audio-Lingual Method): foi desenvolvido durante a Segunda Guerra Mundial, por estruturalistas americanos. A gramática e a estrutura eram o ponto de partida para o aprendizado de determinada língua. Acreditava-se que o aprendizado de uma língua era um processo de formação de hábitos e, por isso, focavase na pronúncia e na intensa repetição de frases padrões. Em função disso, os itens lexicais eram selecionados de acordo com sua simplicidade e familiaridade, para que a repetição (drills) fosse de fácil memorização.

- Método Comunicativo (Communicative Language Teaching): Hymes (1972) apresentou o conceito de competência comunicativa e o definiu como sendo o "conhecimento interno da apropriação situacional da língua". Ou seja, ter competência comunicativa significa usar a linguagem e o vocabulário em função da comunicação. $O$ principal objetivo deste método é proporcionar aos aprendizes da língua contato com a língua alvo e promover fluência (fluency) e proficiência (accuracy). Para Richards e Rodgers (apud ZIMMERMAN, 1997, p. 10), “fazer com que a comunicação seja o objetivo do ensino de uma língua e desenvolver procedimentos para o ensino das quatro habilidades é reconhecer a interdependência da língua e comunicação". Wilkins (apud ZIMMERMAN, 1997, p. 133), um dos precursores da Abordagem Comunicativa, é citado por Paiva (2004), afirmando o seguinte: “aprender vocabulário é aprender como as palavras se relacionam com a realidade externa e como elas se relacionam umas com as outras." Desta forma, o vocabulário deve exercer uma função específica, ser ensinado e utilizado a fim de suprir a necessidade de comunicação, e não isoladamente, como um fim em si mesmo.

- Método Natural (The Natural Approach): este método dá ênfase à compreensão e ao insumo significativo (não necessariamente gramática correta), e o vocabulário é considerado muito importante no processo da aquisição da linguagem, já que, de acordo com este método, "acquisition depends directly on the ability to recognize the meaning of key elements in the utterance. Thus, acquisition will not take 
place without comprehension of vocabulary" ". (KRASHEN; TERREL apud ZIMMERMAN, 1997, p. 131).

Independentemente da abordagem adotada para se ensinar ou aprender a língua alvo, as estratégias para aquisição da língua são de extrema importância, uma vez que se deve considerar o que é necessário e o que não é tão necessário de ser ensinado. $\mathrm{O}$ critério para essa escolha é baseado no fato de que algumas palavras ocorrem com muito mais frequência do que outras, e isso as torna, então, mais relevantes para o aprendizado de determinado idioma. A seguir, serão apresentados alguns princípios para definição de itens lexicais a serem trabalhados bem como algumas estratégias de aquisição de vocabulário na L2.

\section{Estratégias para ensino e aquisição de vocabulário na língua alvo}

Nation (2003) classificou o léxico da Língua Inglesa em high-frequency words (palavras de alta frequência), academic words (termos acadêmicos), technical words (termos técnicos) e low-frequency words (palavras de baixa frequência). As palavras de alta frequência são palavras funcionais como help (ajuda), day (dia), different (diferente), etc. e, segundo o autor, somente com essas palavras é possível estabelecer diálogos cotidianos.

Os termos acadêmicos ocorrem em testes e ambientes acadêmicos, mas também podem ser encontrados na literatura e em jornais que circulam diariamente. Alguns exemplos de termos acadêmicos são abstract (resumo acadêmico), affect (afetar), margin (margem), policy (política), etc. No entanto, esses termos acadêmicos podem se tornar palavras de alta frequência, dependendo do contexto e do público-alvo de um curso de línguas, por exemplo, se em um curso de línguas, grande parte dos aprendizes de uma turma são alunos universitários, os itens lexicais que seriam do grupo de termos acadêmicos, passarão a ser de uso recorrente (ou alta frequência) para estes alunos.

Os ditos termos técnicos são utilizados em áreas específicas do conhecimento e, fora desse contexto, fazem pouco ou nenhum sentido ao aprendiz. É importante destacar que parte do vocabulário técnico também consiste de palavras de alta frequência e de termos acadêmicos. Finalmente, têm-se as palavras de baixa frequência. O que determina a baixa frequência de uma palavra ou grupo de palavras varia de acordo com o contexto. Por exemplo, termos técnicos ou acadêmicos serão de baixa frequência na comunicação cotidiana. Em relação a esse aspecto, McCarthy (1990, p. 80) esclarece 
que utilizar listas baseadas na frequência das palavras pode ocasionar alguns problemas, já que:

[words from the same subject are not necessarily of the same frequency and words of similar frequency come from widely different subject areas; so if you want to organize your vocabulary teaching on a subject basis, then the best we can hope for is somehow to work out intuitively, or through studying a limited set of texts, what the most frequent words are in that subject area. $]^{8}$

Então, a questão que se coloca é: 'como ensinar vocabulário e como auxiliar os alunos a aprendê-lo?' É de conhecimento geral que, apenas por meio de listas com novas palavras, o ensino de vocábulos não é muito eficiente, mas, por outro lado, agrupar e classificar as palavras pode ser o primeiro passo para a aquisição de vocabulário.

Por exemplo, se o conteúdo a ser trabalhado em sala de aula é 'oferecer e pedir alimentos em um restaurante', para melhor memorizar o vocabulário relacionado a esse assunto, uma boa ideia é classificar os itens em aperitivos, pratos principais e sobremesas, ou até mesmo classificá-los em proteínas, carboidratos e gorduras. Se o vocabulário-alvo é frutas, é possível organizá-las em frutas silvestres e cítricas, ou ainda por cores e vitaminas encontradas nessas frutas. Dessa forma, o ensino de vocabulário não parece algo isolado, que possa ser feito com base apenas em uma lista de palavras.

Além do agrupamento de palavras, outras estratégias podem ser utilizadas para a apresentação e aquisição de vocabulário, dentre as quais: desenhos ilustrando o significado das palavras, elaboração de frases com a palavra em contextos diferentes e, sempre que possível, o uso do novo item lexical na fala, ou melhor, nas atividades voltadas à oralidade. Ur (1991) cita algumas maneiras de se apresentar o significado de novos itens lexicais, possibilitando aos aprendizes da língua alvo a sua aquisição, dentre as quais estão:

- Definição concisa (concise definition): constitui a definição concisa da palavra;

- Descrição detalhada (detailed description): consiste em uma descrição mais detalhada do vocábulo, informando sua aparência e/ou suas qualidades, não apenas a definição;

- Exemplos (examples): constitui a apresentação de exemplos do uso da palavra, ou seja, na apresentação de hipônimos (palavras que se relacionam pelo sentido dentro de um conjunto, ligando-se uma a outra por afinidade); 
- Ilustração (illustration): consiste na apresentação do significado da palavra por meio de imagens ou objetos (realia);

- Demonstração (demonstration): compreende demonstrações do significado da palavra por meio de mímicas ou representações;

- Contexto (contexto): consiste na apresentação do contexto em que o vocábulo aparece, como, por exemplo, em uma frase ou texto, para que ocorra a dedução do sentido do item lexical;

- Sinônimos (synonyms): compõe a apresentação de sinônimos do vocábulo desconhecido, por meio de palavras mais simples, que já sejam de conhecimento do aluno;

- Antônimos (opposites): ao contrário da estratégia acima, equivale à apresentação de antônimos do vocábulo desconhecido, por meio de palavras mais simples, que já sejam de conhecimento do aluno;

- Tradução (translation): consiste na tradução direta da palavra para a língua materna;

- Combinações (collocations): constitui a apresentação de combinações possíveis da palavra com determinados verbos ou substantivos.

Essas estratégias podem ser utilizadas tanto pelo professor, ao apresentar um vocábulo novo aos alunos, como pelo próprio aprendiz, ao buscar a melhor forma para memorizar e aprender os novos itens lexicais. A importância do contexto não deve ser negligenciada durante este processo, para que a aquisição não se dê de forma isolada e descontextualizada, ocorrendo de forma menos eficaz ou até mesmo, muitas vezes, não ocorrendo.

Brown (2001) defende a ideia de que o ensino de vocabulário deve ser feito de modo contextualizado e significativo para o aprendiz. Portanto, o ensino de vocabulário baseado em listas, definições ou memorizações é rejeitado pelo autor, que afirma: "rather than isolating words and/or focusing on dictionary definitions, attend to vocabulary within a communicative framework in which items appear. Students will then associate new words with a meaningful context to which they apply". 9

Ainda de acordo com Brown, acerca da aquisição de vocabulário, é importante incentivar os alunos a desenvolverem estratégias para inferir o significado das novas palavras que constantemente se apresentam aos aprendizes. Além disso, o autor argumenta que se deve aproveitar o ensino não planejado de vocabulário, ou seja, 
aqueles momentos em que o aluno pergunta sobre uma palavra que não está no plano, ou quando a palavra aparece e o professor sente que deve dar atenção a ela. A ideia defendida por Brown vai ao encontro da proposta sugerida pelo método comunicativo, no qual o contexto tem um valor primordial para a aprendizagem, e tudo o que o aluno produz é no sentido de realizar determinada situação comunicativa.

Após ter desenvolvido - e, espera-se, ensinado - determinado vocabulário ao longo das aulas, inevitavelmente surge um outro questionamento: 'como saber se os alunos realmente adquiriram aquele vocabulário e quais as estratégias mais utilizadas por eles?' A esse respeito, Ur (2001) esclarece que “there are various reasons why we remember some words better than others: the nature of the words themselves, under what circumstances they are learned, the method of teaching and so on". ${ }^{10}$ No intuito de tentar compreender como se dá a memorização de vocabulário e, consequentemente a sua aquisição, Ur (2001) conduziu um experimento que a levou às seguintes conclusões: os itens lexicais são melhor memorizados e, portanto, adquiridos se trabalhados em sessões espaçadas, ou seja, abordados no início da lição, revisados um pouco mais tarde, na mesma lição, e retomados na próxima lição.

Além disso, a autora concluiu que as pessoas tendem a memorizar mais as palavras com significado pessoal ou emotivo, por exemplo, mom (mamãe), dad (papai), love (amor), sex (sexo) e que, ao se trabalhar com listas de palavras, as que estão no começo da lista tendem a ser melhor memorizadas. Tendo isso em mente, chega-se à conclusão de que as palavras de maior relevância ou frequência devem ser ensinadas antes das demais, ou seja, devem aparecer no topo das listas ou no começo de uma lição.

Além de tentar esclarecer o processo de memorização de vocabulário, Ur (2001) também discute diferentes formas para a avaliação da aquisição de itens lexicais. Segundo a autora, é possível avaliar a dimensão de aquisição de vocabulário, com base nas seguintes atividades, geralmente desenvolvidas em sala de aula:

- Múltipla escolha (Multiple choice): neste tipo de atividade, apenas o sentido denotativo das palavras é testado;

- Relacionar as colunas (Matching columns): esta atividade visa relacionar itens lexicais. Com isso, apenas o significado da palavra é averiguado; 
- Identificar o diferente (Odd one out): trata-se de uma atividade com vista a identificar o item lexical que não pertence ao grupo. Assim, como na anterior, apenas o significado é testado;

- Escrever frases ou sentenças (Writing sentences): tem como fim a elaboração escrita de enunciados. Neste tipo de atividade, apenas a pronúncia não é averiguada. Todavia, não é possível testar precisamente o conhecimento do aluno quanto ao significado da palavra;

- Ditado (Dictation): apenas o reconhecimento por meio da compreensão oral (listening) e a grafia de vocábulos específicos são testados;

- Completar espaços / Preencher lacunas (Gap-filling): este tipo de atividade testa significado, grafia, gramática e colocação do termo. É uma das atividades mais completas para se avaliar a aquisição de vocabulário;

- Tradução (Translation): testa todos os aspectos da língua, mas é difícil, às vezes impossível, encontrar a tradução equivalente para alguns termos de uma língua para outra;

- Completar sentenças (Sentence completion): testa apenas o significado de determinado vocábulo.

No entanto, é importante destacar que a definição proposta por Ur (2001) a respeito de cada uma dessas atividades pode ser discutida a partir da elaboração de cada uma delas. Nem toda a atividade de múltipla escolha trabalhará apenas o sentido denotativo das palavras, uma vez que é possível colocar, como opções, diferentes âmbitos e sentidos conotativos também. A autora expõe, na definição das atividades, um objetivo específico de trabalho com cada uma delas, o que pode ser totalmente modificado, considerando-se contextos específicos de ensino, aos critérios do professor.

Ainda, existe a possibilidade da aquisição de vocabulário por meio da leitura de histórias. Conforme afirma Ferrari (2004), “through stories, learners are often exposed to a large variety of contexts that can be explored orally in different ways in the classroom so as to increase and reinforce their vocabulary". ${ }^{11}$

A autora apresenta a Teoria Conexionista (Connectionist Theory), que prevê o desenvolvimento de vocabulário por meio da prática de leitura e contação de histórias na aula de língua estrangeira. Dessa forma, a aquisição de vocabulário é incidental, pois o aluno é exposto, sem instruções formais, a insumos oferecidos por um determinado contexto linguístico e os conceitos são formados quando há necessidade de 
comunicação. Ainda segundo alguns pesquisadores, a leitura extensiva (extensive reading) é mais eficaz na aquisição de vocabulário do que exercícios de prática mecânicos e isolados.

Por outro lado, é importante ressaltar que Ferrari (2004) menciona a necessidade do ensino formal de vocabulário aos falantes não nativos, para que haja a sua consolidação, além da prática de leitura, conforme já mencionada. Isso porque o processo de aquisição de vocabulário por parte de falantes não nativos é diferente do desenvolvido por falantes nativos, já que para estes ler e entender o uso de determinado termo em um contexto específico seria o suficiente para internalizá-lo. Nesse caso, a leitura e contação de histórias devem ser utilizadas apenas como mais uma estratégia de aquisição do vocabulário.

Também é importante considerar que, para a aquisição do vocabulário, o item lexical precisa ter uma frequência significativa nas aulas, para que o aluno o memorize e adquira. A autora anteriormente citada argumenta que existem níveis intermediários de conhecimento, o que reduz o intervalo entre a compreensão e a produção do vocabulário em si. Assim, a aquisição se dá da seguinte forma:

\section{Recepção $\rightarrow$ Reprodução (com ou sem compreensão) $\rightarrow$ Produção}

Em outras palavras, primeiramente o aprendiz lê ou ouve a palavra pela primeira vez. Esse estágio é definido como Recepção. No próximo estágio - Reprodução - dá-se o reconhecimento do vocábulo, que acontece por meio da recepção repetida. Na Reprodução, o aluno reconstrói o que já ouviu, pode repetir e usar o vocabulário até mesmo sem ter compreendido bem o seu significado. E, por fim, na Produção, o aluno reconstrói o vocábulo de maneira criativa, ou seja, adapta e inventa de acordo com as necessidades e o contexto considerado. É na fase da Produção que o professor consegue avaliar se o vocábulo foi realmente internalizado pelo aprendiz.

Como se pode observar, é bastante difícil selecionar apenas um tipo de atividade para averiguar o que foi realmente aprendido em termos de vocabulário, uma vez que cada atividade visa a contemplar um aspecto específico da língua. Considerando esse aspecto e os demais fatores discutidos neste trabalho sobre aquisição de itens lexicais, pode-se dizer que, depois de definido o vocabulário a ser ensinado, partindo-se da relevância do conteúdo e do interesse do aprendiz, a maneira mais apropriada para 
verificar se o aluno realmente o adquiriu é observando se ele consegue tornar os novos vocábulos significativos nas interações estabelecidas no seu dia-a-dia, utilizando-os de forma contextualizada. Para que isso aconteça, no entanto, é preciso que o professor oportunize situações, dentro ou fora do contexto escolar, de uso desse vocabulário por parte dos aprendizes, de forma que eles possam atingir seus objetivos sociais por meio de sua própria atuação ou performance linguística.

\section{Metodologia}

A presente pesquisa define-se como: bibliográfica, porque busca subsídios teóricos para a questão investigada, ou seja, estratégias adotadas por aprendizes de Inglês como Língua Estrangeira para aquisição de vocabulário; de análise documental, porque propõe uma análise das primeiras cinco unidades do livro didático American English File 2 - adotado para acadêmicos do terceiro semestre do Curso de Letras do Centro Universitário Franciscano/Santa Maria -, a fim de se fazer um levantamento das estratégias de aquisição de vocabulário mais recorrentes nessas unidades; e de campo, de caráter etnográfico e qualitativo, porque faz uso de um questionário para levantamento das concepções dos participantes deste estudo sobre as estratégias de aquisição de vocabulário mais frequentemente utilizadas. A análise dessas concepções revelará equivalência ou não entre as estratégias adotadas pelo livro didático no ensino de vocabulário e as estratégias utilizadas (in)conscientemente pelos aprendizes da língua alvo.

\section{AQUISIÇÃO DE VOCABULÁRIO EM INGLÊS COMO LÍNGUA ESTRANGEIRA}

Instrumento de coleta de dados: Questionário $^{12}$

Nome:

Idade:

Curso:

\section{Primeira parte: Concepções dos aprendizes}

1) Há quanto tempo você está estudando Inglês como Língua Estrangeira (ILE)? 
2) O que você considera mais difícil?

( ) o uso da gramática já estudada

( ) a memorização/aprendizagem de novos itens lexicais

( ) a pronúncia correta das palavras

( ) outro.

Qual?

3) O que você tem feito para ampliar seu vocabulário? Você faz uso de estratégias? Quais? Explique.

\section{Segunda parte: Identificação e avaliação das estratégias}

4) A seguir, serão propostas atividades para reconhecimento dessas estratégias que você utiliza para aquisição de vocabulário:

a) Draw lines connecting the pairs of opposites:

A

Brave

Female

Cheap

Asleep

Fail
B

awake

expensive

succeed

cowardly

male 
b) Underline the odd one out:

$\begin{array}{lllll}\text { Discuss } & \text { Argue } & \text { Fight } & \text { Chat } & \text { Talk } \\ \text { Done } & \text { Went } & \text { Blown } & \text { Found } & \text { Come }\end{array}$

c) For each of the following words, write a sentence that makes its meaning clear:

Abroad:

Slim:

Decision:

d) Find in the paragraph below the words to the following definitions:

1. : good, but not excellent

2. : the place where your legs meet the front of your body

3. : very hot

4. : to behave in a dishonest way

\section{Woman Suspects Husband of Cheating, Pours Boiling Water on His Groin}

Florida: A 52-year old woman has been charged with aggravated battery after she poured boiling water onto her husband's groin. The woman says she scalded her husband because she suspected he was cheating.

The woman was reportedly angered by her spouse's unexplained absence the previous night. Her husband, 51, is in a fair condition in hospital with seconddegree burns.

Fonte: www.shortnews.com 


\section{Resultados e Discussão}

Nesta seção, serão apresentadas e discutidas primeiramente as atividades que são recorrentes no livro didático adotado pelos alunos do terceiro semestre para a aquisição de vocabulário, e em seguida, as estratégias que os alunos utilizam, com base na pesquisa feita com os mesmos.

\section{Análise e identificação das estratégias de aquisição de vocabulário adotas no livro didático American English File 2}

Ao longo desta subseção, será analisado o livro didático adotado pelo terceiro semestre do curso de Letras: Português e Inglês do Centro Universitário Franciscano. Trata-se do American English File 2 - Student book. Durante a análise, são estudadas as diferentes formas pelas quais o vocabulário é apresentado e trabalhado nas Unidades 1 a 5 (unidades que são estudadas no terceiro semestre do Curso). É importante destacar que, durante esta investigação, são analisadas apenas as atividades que são especificamente intituladas como trabalho de vocabulário, não se considerando atividades de Gramática, Leitura e Compreensão, que podem também ser consideradas formas passivas de apresentação de novos vocábulos.

O livro é dividido em nove unidades e cada unidade é subdivida em 4 seções (A, B, C e D), além de uma seção de prática e consolidação das competências trabalhadas ao longo da unidade, uma seção de revisão de toda a unidade, intitulada What do you remember? e, por fim, um banco de atividades em anexo no final do livro para a prática específica de vocabulário e gramática.

A primeira Unidade Who's Who? aborda apresentações pessoais, adjetivos, membros da família e partes do corpo. Na seção 1A, não há trabalho específico sobre vocabulário. Na seção 1B, há trabalho com vocabulário por meio de um exercício de palavras cruzadas (p.6) e, nesta atividade, além de desenvolver a proposta, são feitas atividades de perguntas associativas com ênfase nos opostos (Exemplo: the opposite of thin or slim is....) e interpretação de enigmas (Exemplo: Your sister's daughter is your...). Em seguida, é proposto que o aluno vá ao banco de atividades extras (p.145) para trabalhar um pouco mais com o uso de adjetivos utilizados para definir características pessoais. O vocabulário é tratado da mesma forma: solução de enigmas e opostos, porém sem o uso de palavra cruzada. 
$\mathrm{Na}$ seção $1 \mathrm{C}$, são trabalhadas as partes do corpo. Inicialmente, como atividade introdutória, é apresentada uma obra de Pablo Picasso (Portrait of Dora Maar), para que os alunos a descrevam e rotulem a imagem, que é o rosto e o colo de uma mulher, fazendo uso de termos relativos às partes do corpo, já disponibilizadas no exercício. Em seguida, é proposta mais uma atividade no banco de atividades (p.146), similar à anterior, ou seja, rotulação das partes do corpo.

$\mathrm{Na}$ seção 1D, os alunos são estimulados a definir ou parafrasear termos ou expressões, utilizando-se especialmente dos pronomes relativos who, which, where, that, entre outros. Nesse caso, a atividade proposta é completar algumas definições com verbos e substantivos disponibilizados no próprio exercício. Por exemplo: It's

who works in a restaurant. Nesse caso, a resposta seria somebody. Em seguida, há outra atividade de completar definições. Por exemplo: a tourist is somebody

$\mathrm{Na}$ última seção, a de revisão, o vocabulário é exercitado em três atividades, com objetivos distintos: completar os espaços/as lacunas, marcar o que não pertence ao grupo (Odd one out) e associar ou definir conceitos, por meio de atividades de completar frases e exemplos.

Portanto, na primeira unidade do livro didático analisado, observa-se recorrência quanto ao uso das seguintes estratégias de aquisição de vocabulário: 1)Definir por meio de Opostos; 2) Definir o vocábulo; 3) Rotular por meio de ilustrações; 4) Completar frases; 5) Identificar o vocábulo diferente; e 6) Completar frases.

Na segunda unidade, são apresentados vocabulário e gramática necessários para falar sobre férias e atividades de lazer. Na seção 2A, Right place, wrong time, a primeira atividade é de vocabulário e propõe que os alunos façam um brainstorming das ações que eles gostam de praticar nas férias (In one minute, write down five things you like doing when you're on vacation.) Em seguida, sugere-se aos aprendizes irem ao banco de atividades, já que lá existem duas atividades destinadas à aquisição de vocabulário: uma de identificar/rotular expressões a partir das figuras (Match the verbs and pictures. / Match the words and pictures.) e outra de completar os espaços com verbos e expressões relacionadas a atividades de férias (Complete the verb phrases.).

Na seção 2B, A moment in time, o vocabulário trabalhado é o das preposições in, on, at, por meio de uma atividade de completar os espaços (Complete the sentences with at,in, or on). Além disso, existe uma outra atividade de completar, no banco de atividades (Complete the chart with at, in, or on). 
Na seção 2C, fifty years of pop music, o vocabulário é trabalhado por meio de uma atividade de speaking, na qual os alunos devem utilizar algumas Question Words (In pairs or groups, answer the questions). Na seção 2D, One October evening, phrasal verbs são apresentados em uma atividade de vocabulário. Trata-se de uma atividade de associação por meio da substituição de um verbo simples por uma das expressões de phrasal verbs disponibilizadas na própria atividade (Make verb phrases with a verb from circle 1 and a phrase from circle 2). Na última parte, na revisão, o vocabulário é trabalhado por meio de três atividades de completar espaços (Complete the phrases with a verb/ Complete the sentences with at,in, ot on./ Complete the questions with a question word.)

Portanto, na segunda unidade do livro didático analisado, observa-se recorrência quanto ao uso das seguintes estratégias de aquisição ou aprendizagem de vocabulário: 1) Brainstorming; 2) rotular as figuras; 3) completar espaços; 4) speaking; e 5) associar/substituir vocábulos.

Já na terceira unidade, na seção 3A, Where are you going?, Phrasal Verbs com o verbo look são apresentados por meio de uma atividade de completar espaços (Complete the sentences with for, forward to, or through) e uma atividade de identificação por definição da palavra (Look at Marina's interview on page 28 again. Find and underline three expressions with look. Match them to their dictionary definition.).

$\mathrm{Na}$ seção 3B, The pessimist's phrase book, a primeira tarefa, também com verbos, é feita com base na associação de opostos (What's the opposite of the verbs in $A$ ? Use a verb from B.) e, em seguida, um exercício no banco de atividades, no qual encontra-se outra atividade de associação por opostos, mas também com associação de figuras que ilustram o significado dos verbos (Match the verbs and pictures).

Na 3C, I'll always love you, novamente são apresentados Phrasal Verbs, desta vez com a partícula adverbial back. Nesse contexto, a atividade proposta é de completar espaços, estes inseridos em diálogos curtos (Complete the dialogues with a phrase from the box). E, na seção 3D, I was only dreaming, combinações de verbos e preposições são apresentadas novamente por meio de uma atividade de completar espaços, com a preposição correta, em sentenças específicas (Complete the questions with a preposition from the box). E, por fim, na unidade de revisão, encontra-se mais uma atividade de completar espaços (Complete the sentences with the correct preposition). Além dela, há uma atividade de ligar ou relacionar perguntas/sentenças com suas respectivas respostas 
(Match the phrases) e outra de associar verbos opostos (Write the opposite verb phrase).

Portanto, na terceira unidade do livro didático analisado, observa-se recorrência quanto ao uso das seguintes estratégias de aquisição ou aprendizagem de vocabulário: 1) completar espaços; 2) associar por definição do vocábulo; 3) relacionar os opostos; 4) identificar por meio das figuras; e 5) ligar as sentenças.

A quarta unidade, From rags to riches, dá início ao ensino de vocabulário por meio de uma atividade de leitura e, em seguida, de três atividades de associação de figuras para apresentar o vocabulário referente ao tema vestuário (Match the verbs and pictures). Na seção 4B, Family conflicts, alguns phrasal verbs são trabalhados por meio de uma atividade de completar espaços (Read the magazine article and complete it with these verbs), seguida de uma atividade de memorização (Cover the text. Can you remember the eight bad habits?) e uma de comunicação oral (In pairs, say which of these things are a problem in your home and why. What else is a problem?), a fim de já utilizar o vocabulário abordado ou ensinado.

Na seção 4C, Faster, faster!, o vocabulário é apresentado por meio de uma atividade de leitura, associada a uma atividade de inferência, na qual o aluno precisa adivinhar o significado das palavras destacadas no texto por meio do contexto (Now read paragraphs 1-6. In pairs, look at the highlighted expressions with the word time and "guess" their meaning). $\mathrm{Na}$ seção 4D, The World's friendliest city, são apresentados alguns adjetivos referentes a características ou atributos de cidades por meio de uma atividade de associação por opostos (What are the opposites of these adjectives?). No banco de atividades, encontra-se uma atividade de associar adjetivos a figuras (Match the words and pictures 1-8) e quatro atividades de ligar ou relacionar os opostos (Match these adjectives with their opposites in A./ Cover the words and look at the pictures. Remember the adjectives and their opposites/ Put the adjectives with the correct prefix to make opposites./ test a partner. A say and adjective. B say the opposite.). Na unidade de revisão, são feitas três atividades: duas de completar espaços (Complete the phrases./ Complete the sentences.) e uma terceira de associar os opostos (Write the opposite adjective).

Portanto, na quarta unidade do livro didático analisado, observa-se recorrência quanto ao uso das seguintes estratégias de aquisição ou aprendizagem de vocabulário: 1) 
associar figuras; 2) completar frases; 3) adivinhar significado pelo contexto; e 4) associar opostos.

Na quinta e última unidade analisada, a Unidade 5A, Are you a party animal?, o vocabulário trabalhado é o de verbos seguidos por outros verbos no infinitivo, assumindo a configuração de locução verbal. Não há nenhuma atividade específica voltada à aquisição de vocabulário nesta seção, mas há instrução para que o aluno dirijase ao banco de vocabulário, onde há uma lista de verbos seguidos de infinitivo, o que contribui para a assimilação do uso e da semântica de verbos comumente utilizados na língua alvo. No banco de atividades comunicativas, há um exercício de completar espaços (guess the infinitive).

Na seção 5B, What makes you feel good, repete-se o mesmo tipo de atividades com verbos, porém, nessa etapa, são formas verbais seguidas de gerúndio (-ing). Há uma referência ao banco de vocabulário, onde os alunos encontram novamente uma lista de verbos e uma atividade comunicativa, na qual eles devem escolher alguns tópicos presentes nesta atividade para comentar com um colega (Work in pairs. Choose five things to talk about from the list below). Na seção 5C, How much can you learn in a month?, especificamente na seção de vocabulário, são apresentados os modificadores (modifiers), os quais são trabalhados por meio de uma atividade de completar espaços (Complete the chart with the words in the box).

$\mathrm{Na}$ seção 5D, The name of the game, o vocabulário apresentado refere-se a esportes e preposições de movimento, trabalhados logo no início da seção, juntamente com uma atividade de speaking. Primeiramente, há uma atividade de categorização dos esportes em colunas, de acordo com os verbos que os acompanham (Look at the photos. Can you name the sports? Write them in the correct columns). Após, são propostas duas atividades de conversação sobre esportes, para serem feitas em pares ou duplas (In pairs, say.../ Ask and answer with a partner.). Em seguida, é proposta uma atividade de completar espaços, juntamente com associação de figuras, para trabalhar as preposições (Where did the ball go? Complete with a preposition.). Finalmente, no banco de vocabulário, verifica-se que há mais duas atividades voltadas ao ensino e à aquisição de vocabulário: uma de completar os espaços com a preposição correta e outra de associar as preposições com as imagens expostas no livro (Complete the chart with at, in, or on/ Match the prepositions and pictures). Na unidade de revisão, observa-se que há mais 
três atividades de completar frases (Complete the sentences/ Complete the sentences with a preposition/Complete the sentences with play, do and go in the correct form).

Portanto, na quinta unidade do livro didático analisado, observa-se recorrência quanto ao uso das seguintes estratégias de aquisição ou aprendizagem de vocabulário: 1) exposição de exemplos; 2) comunicar-se utilizando o vocabulário apresentado; 3) completar frases; 4) categorizar os vocábulos e 5) associar figuras.

Por fim, com base na análise das estratégias de aquisição de vocabulário adotadas pelo livro didático American English File 2, observa-se que há predomínio da estratégia 'completar espaços', uma vez que a mesma aparece em três das cinco unidades analisadas. Assim sendo, percebe-se que, apesar de o livro mencionado ser definido como um material de ensino baseado no método comunicativo, ao se tratar do trabalho com vocabulário, especificamente, as atividades são, na sua maioria, de caráter Behaviourista, indo ao encontro, portanto, do método Audiolingual.

\section{Análise e identificação das estratégias de aquisição de vocabulário utilizadas pelos acadêmicos do curso de letras do terceiro semestre}

Nesta subseção serão analisadas as estratégias de aquisição de vocabulário geralmente utilizadas pelos aprendizes pesquisados, conforme as respostas fornecidas ao questionário que lhes foi aplicado. A análise parte do depoimento de nove acadêmicos do terceiro semestre do curso de Letras, dentre os quais, grande parte já estuda a Língua Inglesa como segunda língua há uma média de três a quatro anos.

Verificou-se, primeiramente, que oito dos nove alunos que responderam ao questionário apontaram a memorização e aprendizagem de novos itens lexicais como sendo seus maiores obstáculos no processo de aprendizagem de língua inglesa. Num segundo momento, constatou-se que, dentre as principais estratégias utilizadas pelos acadêmicos para identificação e aprendizagem de novos vocábulos, estão: 1) assistir a filmes (com legendas em português ou inglês); 2) escutar e traduzir letras de música ou textos em geral; e 3) ler textos ou livros em inglês.

Além dessas estratégias, apontadas pelos participantes do estudo, existem outras possibilidades destinadas à aprendizagem de língua inglesa, tais como: escrever textos na língua alvo, utilizar o dicionário sempre que necessário, fazer exercícios voltados à aprendizagem do idioma, adivinhar o significado de alguns termos em inglês, 
considerando-se o contexto, com base em inferências e associações a outros idiomas, tais como o alemão.

Nesse caso, verifica-se que a leitura é o mecanismo mais utilizado pelos aprendizes para a aquisição de vocabulário. Desse modo, uma vez que, na leitura, os novos itens lexicais estão inseridos num dado contexto, a contextualização acaba sendo a estratégia que parece mais auxiliar os aprendizes da língua alvo na identificação do(s) significado(s) de determinado termo ou item lexical. Esse resultado remete novamente às considerações de Brown (2001) acerca do ensino e aprendizagem de vocabulário em língua estrangeira, que considera o contexto como elemento primordial para a aquisição de determinado idioma.

\section{Considerações finais}

Sabe-se, então, que, para que um aprendiz obtenha sucesso na aprendizagem de uma segunda língua, o conhecimento de vocabulário é fundamental. Para que o mesmo alcance esse objetivo, existem muitos parâmetros a serem considerados. O papel do professor, durante este processo, é de extrema importância, uma vez que ele deve ter conhecimento sobre as diferentes metodologias de ensino e, além disso, como o vocabulário é abordado em cada uma, para que seja capaz de fazer a escolha correta de como apresentar os vocábulos, de acordo com as necessidades do aprendiz.

Portanto, para o desenvolvimento da competência linguístico-comunicativa, conforme mencionado na seção de 'Introdução' deste estudo, é necessário que o aprendiz, futuro profissional, adquira um número considerável de vocábulos, indispensáveis à comunicação, e, além disso, saiba fazer uso de estratégias específicas, de forma adequada, ao ensiná-los. Em função disso, o emprego de estratégias de aquisição de vocabulário em língua inglesa é relevante para o desenvolvimento dessa competência, que acaba por caracterizar o perfil do profissional da área.

$\mathrm{Na}$ análise do material didático, parte do corpus deste estudo, verificou-se grande recorrência de atividades cujo objetivo é associar significados e completar sentenças, o que remete ao método audiolingual de trabalho com vocabulário. Já na análise do instrumento de coleta de dados, aplicado aos participantes deste estudo, verificou-se que a leitura é a estratégia mais utilizada para aquisição de vocabulário dentre os aprendizes da língua alvo, o que remete ao Método Comunicativo e à Teoria Conexionista, conforme explicitados anteriormente. 
Nesse caso, percebe-se que o contexto é o principal fator envolvido na aquisição de vocábulos, seja por meio de sentenças isoladas, textos, ou letras de música, o que vai ao encontro do que Brown (2001) afirma: que o vocabulário deve ser sempre apresentado dentro de um contexto particular, portanto, de forma bastante significativa, para que o aprendiz veja como o novo item lexical se aplica em determinada situação e, a partir daí, se aproprie do mesmo. Esse princípio está de acordo com os pressupostos do Método Comunicativo, conforme discutido anteriormente, e é citado por Ur (1991) como uma das principais estratégias de apresentação de novos vocábulos aos aprendizes de Inglês como Língua Estrangeira.

\section{Referências bibliográficas}

ALMEIDA FILHO, J.C.P. (2000). Crise, transições e mudança no currículo de formação de professores de línguas. In: FORTKAMP, M. B. M. (Org) Aspectos da linguística aplicada: estudos em homenagem ao professor Hilário Inácio Bohn. Insular: Florianópolis, 2000.

BROWN, D. Teaching by principles: an interactive approach to language pedagogy. $2^{\text {nd }}$ Edition. Longman: San Francisco, 2001.

FERRARI, M. Vocabulary development in English as a Foreign Language through reading and storytelling. In: SARMENTO, S.; MÜLLER, V. (Orgs.). O ensino do inglês como língua estrangeira: estudo e reflexões. Porto Alegre: APIRS, 2004.

HUCKIN, T. Second language vocabulary acquisition. Cambridge: Cambridge University Press, 1997.

HYMES, D.H. (1972). On communicative competence. In: Pride, J.B.; Holmes, J. Sociolinguistics: selected readings. Harmondsworth: Penguin. 1972, p. 269-293.

LEFFA, V. Aspectos externos e internos da aquisição lexical. In: LEFFA, V. J. (Org.) As palavras e sua companhia. Pelotas: ALAB e Editora da Universidade Católica de Pelotas, 2000. p. $15-44$.

McCARTHY, M. Vocabulary. Oxford University Press: Oxford.1990.

NATION, P. Como estruturar o aprendizado de vocabulário. SBS Editora: São Paulo, 2003.

NATION, P; NEWTON, J. Teaching vocabulary. In: COADY, J.; HUCKIN, T. Second language vocabulary acquisition. Cambridge: Cambridge University Press, 1997.

PAIVA, V. L. M. O. Ensino de vocabulário. In: DUTRA, D. P.; MELLO, H. A gramática e o vocabulário no ensino de inglês: novas perspectivas. Belo Horizonte: Faculdade de Letras/UFMG, 2004.

RICHARDS, J. C.; RODGERS, T. S. (1986). Approaches and methods in language learning. Cambridge: Cambridge University Press, 1986. 
SELIGSON, P.; OXENDEN, C.; TATHAM-KOENIG, C. American English file 2. Oxford: Oxford University Press, 2008.

UR, P. A course in language teaching: practice and theory. Cambridge: Cambridge University Press, 1991.

WILKINS, D. A. Linguistics in language teaching. London: Edward Arnold, 1972.

ZIMMERMAN, C. B. Historical trends in second language vocabulary instruction. In: COADY, J.; HUCKIN, T. Second language vocabulary acquisition. Cambridge: Cambridge University Press, 1997.

\section{Notas:}

${ }^{1}$ Professora de Letras: Português e Inglês. Especialista em Metodologias de Ensino de Língua Inglesa pelo Centro Universidade Franciscano (UNIFRA/RS).

ariane_peronio@hotmail.com

${ }^{2}$ Professora do curso de Letras: Português e Inglês do Centro Universitário Franciscano (UNIFRA/RS). Mestre em Estudos Linguísticos pela Universidade Federal de Santa Maria (UFSM/RS). Doutoranda em Estudos Linguísticos pela Universidade Católica de Pelotas (UCPel/RS).

gabrielamarzari@gmail.com

${ }^{3}$ Segundo Almeida Filho (2000), a competência linguístico-comunicativa é aquela que permite ao profissional do ensino proporcionar experiências válidas aos aprendizes na língua alvo.

4 “embora a língua seja feita de palavras, nós não falamos em palavras, mas em sentenças (...)” (Tradução nossa)

5 “interação é o coração da aquisição natural da linguagem” (Tradução nossa)

6 "Pela primeira vez, o vocabulário foi considerado um dos mais importantes aspectos do aprendizado da segunda língua e uma das prioridades no desenvolvimento de uma base científica para selecionar o conteúdo léxico dos cursos de línguas.” (Tradução nossa)

7 “aquisição depende crucialmente do input compreensível. E compreensão é diretamente dependente da habilidade de reconhecer o significado dos elementos chave no ato de dizer (utterance). Assim, a aquisição não acontecerá sem compreensão do vocabulário" (Tradução nossa)

${ }^{8}$ (...) palavras do mesmo campo semântico não são necessariamente da mesma relevância (frequência) e palavras com a mesma relevância vêm de diversos campos semânticos, então, ao organizar o conteúdo de vocabulário a ser ensinado baseado em um tópico, o melhor é fazê-lo intuitivamente, ou estudando em um número limitado de textos, quais são as palavras mais frequentes neles. (Tradução nossa)

9 “(...) é preferível, ao invés de trabalhar com palavras isoladas e definições do dicionário, trabalhar com o vocabulário no contexto comunicativo no qual ele está inserido. O aluno então associará novas palavras em um contexto significativo no qual eles podem aplicá-las.” (Tradução nossa)

10 “(...) existem diversas razões pelas quais lembramos algumas palavras mais do que outras: a natureza da palavra propriamente dita, sob quais circunstâncias ela foi aprendida, o método de ensino e assim por diante". (Tradução nossa)

11 "por meio das histórias, os aprendizes são frequentemente expostos a uma grande variedade de contextos que podem ser explorados oralmente de diferentes formas na sala de aula a fim de aumentar e reforçar seu vocabulário" (tradução nossa).

12 A elaboração deste questionário deu-se com base na obra A Course in Language Teaching, de Ur (1997). 